\title{
SANTA ROSA DE COPÁN; UNA PROPUESTA HACIA UNA NUEVA GESTIÓN DEL AGUA ${ }^{1}$
}

\author{
Autores: \\ Jensen Mauricio Bautista-Perdomo, Nadia Martínez-Villegas
}

\begin{abstract}
Resumen
El acceso a servicios de agua potable y alcantarillado adecuados constituye una condición esencial para la dignidad de las personas y para viabilizar el pleno desarrollo de sus capacidades. En la ciudad de Santa Rosa de Copán, el acceso a estos servicios se encuentra muy limitado, y la escasez de agua, mantiene a la población bajo condiciones de inseguridad sanitaria. En el presente artículo se realizó un análisis de la gestión para el abastecimiento de este líquido, su relación que con la sociedad y el ambiente y la perspectiva económica, reflejado en la necesidad de un sistema de tarifas basada en el consumo. Bajo este concepto, se buscaría que cada usuario pague lo que consume, para crear una nueva cultura en el uso racional del agua.
\end{abstract}

Palabras claves: Santa Rosa de Copán, agua, bien económico, Empresa Municipal Aguas de Santa Rosa, manejo de agua.

\footnotetext{
${ }^{1}$ Los manuscritos aceptados son Artículos en Prensa que han sido revisados por pares y aceptados para su publicación por el Consejo Editorial de esta revista. Todavía no han sido editados para su copia y / o formateados en el estilo de casa de publicación del IIES, y pueden no tener la funcionalidad completa del IIES, por ejemplo, es posible que los archivos suplementarios todavía deben añadirse, así como que los enlaces a referencias no funcionan aún, etc.. El texto podría todavía cambiar antes de la publicación final.

Aunque los manuscritos aceptados no tienen todos los detalles bibliográficos disponibles aún, ya pueden ser citados con el año de la publicación en línea de la siguiente manera: autor (s), título del artículo, la publicación (año). Por favor consulte estilo de referencia de la revista para el orden exacto de estos elementos, abreviatura de nombres de revistas y uso de puntuación.
} 


\title{
SANTA ROSA DE COPAN: TOWARDS AN INTEGRAL MANAGEMENT OF WATER
}

\begin{abstract}
Access to safe water and adequate sewage treatment is critical to the dignity of people to allow the development of their capabilities. At Santa Rosa de Copan, in Honduras, the access to these services is limited, and the scarcity of water keeps the population under unhealthy security conditions. In this paper, we analyzed the current framework of water management at Santa Rosa de Copán and the relationship that would exist among the society, environment, and economy, which prompted us to propose a novel point of view based on tariffs based on consumption. Under this concept, each user would pay and receive what they consume, promoting a full new water culture.
\end{abstract}

Keywords: Santa Rosa de Copan, water, economic value, water management.

\section{El agua: un bien social, económico y ecológico.}

Históricamente el agua ha sido considerada como un recurso natural, ilimitado y renovable. Ha existido cierto consenso en considerar al agua como un bien libre, no económico y, por tanto, gratuito. Sin embargo, el rápido crecimiento de la población y del desarrollo económico en el último siglo está provocando su escasez relativa en muchas zonas. En la actualidad, por otro lado, se reconoce que el agua dulce es un recurso escaso y cuya gestión debe hacer frente a elevados costes, por lo que su gestión adquiere un carácter no sólo social sino también económico. En la declaración de Dublín sobre el Agua y el Desarrollo Sostenible (1992) se menciona en el principio No. 4 que "El agua tiene un valor económico en todos sus diversos usos en competencia a los que se destina y deberá reconocerse como un bien económico. La ignorancia, en el pasado, del valor económico del agua ha conducido al derroche y a la utilización de este recurso con efectos perjudiciales para el medio ambiente. La gestión del agua, en su condición de bien económico, es un medio importante de conseguir un aprovechamiento eficaz y equitativo y de favorecer la conservación y protección de los recursos hídricos.” 
La falsa concepción del ambiente y de los recursos naturales dentro de la categoría de bienes libres define una equivocada relación sociedad-naturaleza y se constituye en uno de sus principales factores de degradación y agotamiento (Avendaño, 2004, citado por Díaz-Pulido et al., 2009). Por otro lado, el tratamiento del agua como un bien económico contribuye a la mejora de las problemáticas de escasez ofreciendo teorías, políticas, principios y estrategias, como la teoría de variación de mercados, la teoría financiera, la política fiscal, el principio de regulación del mercado y la estrategia de fijación de precios, útiles en la integración de políticas de gestión del agua. Martins, Cruz y Barata (2013) menciona que la eficiencia económica para las tarifas de agua potable debe ser iguales a los costos marginales. Ueda y Moffat (2013) diseñan un modelo de tarifa en Inglaterra, donde la medición es opcional y está en relacional proporcional al valor catastral, y demuestran teóricamente que cuando mayor sea el valor catastral, más probable es que el hogar opte por la medición; y menor será el consumo de agua. MartínezEspiñeira (2003) para fijar el precio de una tarifa teórica de agua, realizan un análisis usando la determinación de tarifas por bloque.

\section{El agua: un bien casi exclusivamente social en Santa Rosa de Copán}

Santa Rosa de Copán no se escapa a la problemática de la escasez de agua y al igual que en otras partes del mundo, en Santa Rosa de Copán, su gestión hace frente a elevados costes. Uno de estos es el relacionado con el consumo de energía eléctrica que, en 2012, ascendía a más de L 20,000,000 (Canelo, 2013). Lo anterior, debido a un gasto de operación mayor que el ingreso que recaudaba la empresa operadora de agua, Empresa Municipal Aguas de Santa Rosa. Ingreso que establece el departamento del área comercial de la empresa de aguas, en relación al valor catastral de los bienes inmuebles (Municipalidad de Santa Rosa de Copán, 2015), sin relación alguna con el consumo o el valor de producción, lo que invita a reconsiderar al agua como un bien no sólo social sino también como un bien económico, cuyo valor depende, entre muchos otros, del gasto de electricidad, los productos químicos empleados, el personal técnico, etc.

Santa Rosa de Copán, una propuesta hacia la gestión social y económica del agua. 
De acuerdo al censo del Instituto Nacional de Estadística, Santa Rosa de Copán está experimentando un creciente incremento de población urbana, misma que aumentó de 39,001 habitantes, en el año 2001, a 64,094 habitantes, en el año 2014. Pero ¿qué problemas ocasiona este crecimiento poblacional? Dicho crecimiento ha traído consigo una mayor demanda de agua por parte la población, tanto para las actividades domésticas como para las productivas, trayendo, a su vez, la escasez del recurso. Para el año 2013, la demanda de agua en Santa Rosa de Copán era de 2,656,051 galones $\left(10,061 \mathrm{~m}^{3}\right)$ por día, mientras que la oferta ascendía a tan sólo 1,494,120 galones $\left(5,660 \mathrm{~m}^{3}\right)$ por día (Canelo, 2013). De lo anterior, se origina un déficit en el servicio de agua de un $44 \%$, mismo que se estima que incrementa a razón de $2 \%$ anual, en promedio (Canelo, 2013). En este sentido ¿qué sería necesario para lograr el desarrollo y crecimiento sostenible en Santa Rosa de Copán?. Para lograr un desarrollo y crecimiento de manera sostenible, se necesitaría optimizar los procesos de extracción, distribución y aprovechamiento del agua de una manera económicamente viable y ambientalmente sustentable (Díaz-Pulido et al., 2009). Lo anterior constituye entonces una premisa indispensable para la ciudad de Santa Rosa de Copán. Así, una revaloración del agua, que incluya una visión económica, es necesaria para el suministro suficiente de agua a la población, a través de un mejor funcionamiento de la empresa. Para logar dicho funcionamiento, es primeramente necesario analizar, cuáles son los problemas de captación de agua en Santa Rosa de Copán, cómo agravan estos la escasez y qué mejoraría la oferta para poder satisfacer la demanda. Así, en objetivo de este trabajo es describir algunos de los problemas de captación de agua de Santa Rosa de Copán y su escasez y proponer un nuevo sistema tarifario basado en el consumo. Para lo anterior se realizó un análisis descriptivo de algunos elementos que influye en la crisis de escasez de agua de la ciudad.

En la actualidad, el sistema de captación de agua potable en Santa Rosa de Copán está compuesto por dos fuentes superficiales, Río Higuito y microcuenca La Honduras, y por 8 pozos de extracción de agua subterránea. De estas fuentes, Río Higuito comenzó a funcionar en el 2005 , con tres estaciones de bombeo y una capacidad de 1200 galones por minuto, en el que el agua era elevada a $700 \mathrm{~m}$ de altura a través de una línea de bombeo de aproximadamente $8 \mathrm{~km}$ (Empresa Municipal Aguas de Santa Rosa, 2015). No obstante, a partir del 2006, las galerías de infiltración dejaron de funcionar por las características geológicas de la región y las altas precipitaciones pluviales. El problema era que el sedimento arrastrado por el río obstruía la 
entrada de agua a la primera estación, lo que forzó a la remoción de las galerías y, por consecuencia, a la entrada de agua rica en sedimento a la cisterna de almacenamiento de agua, problema que posteriormente exacerbó operación del proyecto y conllevó a altos costos de operación y mantenimiento del Río Higuito, haciendo inviable la operación continua de este proyecto y, por ende, impactando en la escasez que la población experimenta a través del racionamiento.

En lo que se refiere a Microcuenca La Hondura, ésta es una fuente superficial con una capacidad de hasta 350 galones por minuto en el invierno, que, en comparación con Río Higuito, suministra sólo marginalmente a la población, suministro que se exacerba en el verano a tan sólo 90 galones por minuto por las condiciones climáticas (Empresa Municipal Aguas de Santa Rosa, 2015), lo que contribuye, de una manera importante, al problema de escasez de agua por el relativamente bajo suministro de agua y la variabilidad de éste a lo largo del año. Por otro lado, los pozos subterráneos se encuentran sobre un acuífero de origen volcánico, fracturado, con problemas inherentes de abatimiento cuya recuperación piezométrica deriva en la operación intermitente del bombeo de los pozos, de los cuales no se conocen las características, ni el grado de fracturamiento del mismo y, por ende, tampoco la capacidad de almacenamiento del acuífero, lo que impide la toma de decisiones fundamentadas en la gestión del agua.

Independientemente del suministro o costo del agua, para inicios del año 2015, la mora a la Empresa Municipal Agua de Santa Rosa era del 59.26\% (Empresa Municipal Aguas de Santa Rosa, 2015) y el abastecimiento a la población no era regular, lo que obligaba (y sigue obligando) a una familia promedio a comprar aproximadamente L 4,200 al año por agua cruda, no necesariamente potable, para poder cubrir la carencia de recurso, sin contar la tarifa mensual que deben pagar por agua potable. Interesantemente, el problema tarifario del agua en Santa Rosa de Copán, no es reciente, sino que data de 1999, con el incremento de tarifas y creación de un área comercial para poder aplicarlas, tarifas basadas en función de la renta del inmueble y no en el consumo efectivo. Tarifas que, a pesar del aumento, siguen siendo económicas. Bajo este contexto, en Santa Rosa de Copán, sería deseable un nuevo sistema tarifario, basado, entre otros, en el consumo de agua que la considere no sólo como un bien social sino también como un bien económico; un sistema de administración en donde el agua tenga un precio por unidad que resulte fundamental y, a su vez, promueva el consumo responsable del agua y una nueva 
cultura de uso del agua. Implementar un precio por unidad de volumen generaría e incentivaría la inversión de proyectos alternos, estudios del acuífero y estudios ambientales que actualmente no existen. Adicionalmente, la administración planeada del recurso sería beneficiosa para la sostenibilidad del recurso hídrico (Liu, Shao, Wang, y Hou, 2012).

Por otro lado, administrativa y jurídicamente la Empresa Municipal Aguas de Santa Rosa se encuentra frente a un proceso de desconcentrar y descentralizar el servicio de agua potable y alcantarillado de la Municipalidad de Santa Rosa de Copán. Se espera que esto tenga una incidencia de primera magnitud en la concepción de los procedimientos de estructuración y operación de los sistemas de abastecimiento de la ciudad. Para tal efecto, se tienen que involucrar dos aspectos en la transformación de la Empresa Municipal Aguas de Santa Rosa: la descentralización y la apertura a la participación ciudadana (Ley de Municipalidades, Decreto $\mathrm{N}^{\circ}$ 134-90, publicada en el Diario Oficial La Gaceta del día 29 de octubre de 1990, Ley Marco del Sector Agua Potable y Saneamiento, Decreto No 118-2003, publicada en el Diario Oficial La Gaceta del día 8 de octubre de 2003, Corporación Municipal de Santa Rosa de Copan, 2005. Libro de Actas Municipales). El proceso de descentralización y la apertura a la participación ciudadana han comenzado y está en proceso de consolidarse, mientras tanto, los participantes se encuentran trabajando activa y estrechamente en la concepción de los nuevos procedimientos de estructuración y operación de los sistemas hidráulicos en Santa Rosa de Copán. En la actualidad, prevalece la esperanza de cambios radicales que afecten de manera positiva el abastecimiento de agua a la población.

Determinar el precio del agua potable no es una tarea sencilla, en el sistema tarifario, diferentes elementos pueden incidir en el costo; las opciones tecnológicas (sistemas de bombeo, aprovechamiento de energía alternativas-ejemplo paneles solares), opciones en materia de gestión, perdidas por fugas, recuperación de las deudas, la participación de la población, por ejemplo, reparaciones en fugas de agua en sus casas y uso de tecnologías ahorradoras del recurso, etc. Determinar el precio del agua se vuelve aún más complicado sí se le considera como un bien económico a través del mercado (oferta y demanda), tal y como se menciona en la teoría neoclásica: el análisis de las determinantes de la tasa de regeneración, no se reduce a precios y tasas de descuentos, ni tampoco se le da una eficiente asignación a través del mercado (Blaug, 2001). Dicho problema es complejo ya que la tasa de regeneración se ve afectada por el 
ciclo hidrológico y las partes que lo afectan. No obstante, dentro de las variables que se pueden modificar para mejorar la oferta y suplir la demanda de agua, con una gestión sustentable del servicio, están las siguientes variables.

\section{Variables a modificar en función a la oferta del servicio de agua}

Para ofrecer una mejor oferta de agua en cantidad y calidad, la empresa Municipal Aguas de Santa Rosa tendría que enfocar esfuerzos e inversión en lo siguiente:

o Incrementar el sistema de represamiento en la microcuenca La Honduras.

- Recuperar fuentes superficiales de agua contaminada, como quebradas y arroyos, previa remediación de las mismas.

- Realizar estudios del acuífero de Santa Rosa para conocer su capacidad de almacenamiento y extracción y para proyectar perforaciones sin impactar negativamente el acuífero.

- Construir estructuras de almacenamiento de agua potable como, por ejemplo, tanques, para incrementar la capacidad de almacenamiento y distribución.

- Encontrar nuevas fuentes sustentables de agua.

o Minimizar las fugas.

o Promover la incorporación de nuevas tecnologías ahorradoras de agua en los hogares.

\section{Variables a modificar en función a la demanda del servicio de agua}

"Hasta la década de 1970, el abastecimiento de agua en las ciudades se basaba principalmente en políticas orientadas hacia la oferta, es decir en construir la infraestructura necesaria para obtenerla y así satisfacer las necesidades de la población" (Baumann, 2005, Citado por Salazar y Pineda, 2010). No obstante, hoy en día, también se tiene que dar importancia a la demanda por lo que para satisfacer mejor la demanda de la población, la empresa Municipal Aguas de Santa Rosa tendría que enfocar esfuerzos e inversión en lo siguiente:

- Medir el consumo de agua por propiedad. La micromedición del agua permitiría a la Empresa de Aguas de Santa Rosa la implementación de tarifas basadas en unidades de volumen consumido y no en el valor catastral de las propiedades. Este nuevo sistema 
tarifario, basado en el cobro del servicio de agua por unidad de volumen consumido, promovería, inevitablemente, el ahorro del recurso entre la población.

o Desglosar e independizar el cobro del agua de otros servicios municipales como tren de aseo, bomberos y la red de drenaje. Actualmente dichos servicios están incluidos en un solo recibo de pago, emitido por la Empresa Municipal Aguas de Santa Rosa, lo que ocasiona el descontento generalizado de la población porque la gente piensa que paga un alto costo de agua por un servicio muy limitado, cuando en realidad una parte importante de dicho costo es para otros servicios. En el nuevo sistema propuesto, otros servicios no deberían aparecer. Idealmente, el recibo de pago de la Empresa de Aguas de Santa Rosa sólo deberá incluir el servicio de agua potable.

- Satisfacer la demanda y continuidad del servicio de agua potable a la población de Santa Rosa de Copán en crecimiento.

Santa Rosa de Copán es una ciudad en constante crecimiento y un suministro adecuado a la demanda de agua atraería una mayor inversión y, por ende, un crecimiento más sostenible de la población.

\section{Conclusión}

Santa Rosa de Copán afrenta un problema de captación y escasez de agua que origina un déficit del $44 \%$ respecto a la demanda del recurso. Dichos problemas se encuentra fuertemente afectados por los altos costos del proyecto Río Higuito, la variabilidad estacional de agua en Microcuenca La Hondura, el desconocimiento de las características del acuífero de origen volcánico y su intermitente operación, así como por una facturación inconexa del costo del agua y una importante mora en el retraso de los pagos por parte de la población.

La implementación de un nuevo sistema de tarifas por consumo de agua potable basado en unidades de volumen consumidas permitiría la captación de recursos económicos, en relación a la demanda, para poder cubrir los costos, corrientes y periódicos, de mantenimiento de las fuentes de abastecimiento, la amortización de los equipos, las reparaciones y el mantenimiento de los sistemas de distribución, así como la inversión en estudios de agua subterránea y fuentes superficiales de abastecimiento. La implementación de este nuevo sistema sería de beneficio mutuo tanto para la Empresa Municipal Aguas de Santa Rosa para ofrecer un mejor servicio y para la población que recibiría un servicio más continuo. 


\section{Bibliografía}

Blaug, Mark., (2001). Teoría económica en retrospección. México, D.F. Fondo de Cultura Económica.

Canelo, Noe. I., (2013). Análisis de la capacidad de represamiento de agua en la microcuenca La Hondura como una alternativa para cubrir el déficit proyectado a 10 años del sistema de agua potable de la ciudad de Santa Rosa de Copán. Municipalidad de Santa Rosa de Copan, Empresa Municipal Aguas de Santa Rosa.

Corporación Municipal de Santa Rosa de Copan, 2005. Libro de Actas Municipales

Declaración de Dublín sobre el Agua y el Desarrollo Sostenible (1992). Recuperado febrero 2015. http://www.wmo.int/pages/prog/hwrp/documents/espanol/icwedecs.html

Díaz-Pulido, A. P., Chingaté-Hernández, N., Muñoz-Moreno, D. P., Olaya-González, W. R., Perilla-Castro, C., Sánchez-Ojeda, F. \& Sánchez-González, K. (2009). Desarrollo sostenible y el agua como derecho en Colombia. Revista Estudios Socio-Jurídicos, 11(1) 84-116. Recuperado de http://www.redalyc.org/articulo.oa?id=73311610005

Empresa Municipal Aguas de Santa Rosa (2015). Comunicación personal 21 de febrero

Empresa Municipal Aguas de Santa Rosa (2015). Infraestructura, obras de captación pozos y otros. Recuperado febrero 2015 de http://www.aguasdesantarosa.com/infraestructura/obras-decaptacion-pozos-y-otros

Empresa Municipal Aguas de Santa Rosa (2015). Infraestructura, sistema de agua potable Santa $\begin{array}{lllll}\text { Rosa de } & \text { Copán. } & \text { Recuperado } & \text { febrero }\end{array}$ http://www.aguasdesantarosa.com/infraestructura/redes-de-distribucion

Heinz, I. A., Pulido-Velázquez, M. A., Lund, J.R., Andreu, J., (2007). Hydro-economic Modeling in River Basin Management: Implication and Applications for the European Water Framework Directive. Water Resources Management, Vol. 21, pp. 1103-1225. doi: 10.1007/s11269-006-9101-8

Instituto Nacional de Estadistica, (s.f.). Estimación de la población de Copán por municipio, área urbana y rural, sexo y grupo de edad especiales. 
Ley de Municipalidades, Decreto $N^{\circ} 134-90$, publicada en el Diario Oficial La Gaceta del día 29 de octubre de 1990

Ley Marco del Sector Agua Potable y Saneamiento, Decreto No $118-2003$, publicada en el Diario Ofícial La Gaceta del día 8 de octubre de 2003

Liu, C., Shao, M., Wang, Y., Hou, X., (2012). Status quo of water science \& technology development in china and abroad. Water science \& technology in China: A roadmap to 2050. pp. 37-52. Doi: 10.1007/978-3-642-23632-7_3

Lopez-Mayan, C., (2014). Microeconometric Analysis of Residential Water Demand. Environmental and Resource Economics. Vol. 59, pp. 137-166. doi: 10.1007/s10640-013-97214

Martínez-Espiñeira, R., (2003). Estimating Water Demand under Increasing-Block Tariffs Using Aggregate Data and Proportions of Users per Block. Environmental and Resource Economics. Vol. 26, pp. 5-23. doi: 10.1023/A:1025693823235

Martins, R., Cruz, L., Barata, E., (2013). Water Price Regulation: A review of Portuguese Tariff Recommendations. Public Organization Review. Vol. 13, pp. 197-205. doi: 10.1007/s11115013-0230-2

Salazar A. A, y Pineda P., N., (2010). Factores que afectan la demanda de agua para uso doméstico en México.Región y Sociedad, XXII(49) 3-16. Recuperado de http://www.redalyc.org/articulo.oa?id=10215725001

Ueda, T., Moffatt, P. G., (2013). A Socially Efficient Water Tariff Under the English Optional Metering Scheme. Environmental and Resource Economics. Vol. 54, pp. 495-523. doi: 10.1007/s10640-012-9603-1 